\title{
Smartphone CanSat for Actualization of Real-time Streaming Video Calls Using Remote Screen Touch System with Shape Memory Alloy Actuator*
}

\author{
Hyun-Ung OH, ${ }^{\dagger}$ Hye-In Kim, Jeong-Ki Kim, Jae-Seop CHOI, and Su-Hyeon Kim \\ Space Technology Synthesis Laboratory, Department of Aerospace Engineering, Chosun University, Gwangju 501-759, Korea

\begin{abstract}
This paper reports the Smart Call from the Sky (SCSky) Can Satellite (CanSat) platform developed by Chosun University, South Korea, with a focus on a smart material of shape memory alloy actuator applications. The primary objective of this study is to verify the effectiveness of a remote screen touch system using shape memory alloy wires. The system is currently being utilized to operate the on-board smartphone of a CanSat by telecommands from a ground station. It provides real-time streaming video showing the internal and external states of CanSat through video calls during actual flight. The secondary objective of the mission is to acquire a wide-scan image from the on-board USB cameras, whose elevation angles are actuated using shape memory alloy springs. The effectiveness of the design proposed was validated experimentally through actual flight tests using model rockets.
\end{abstract}

Key Words: CanSat, Shape Memory Alloy, Smartphone, Remote Screen Touch System, Wide-Swath Scan Camera

\section{Introduction}

Can satellites (CanSat) have become practical educational tools for simulating satellite missions through the designing, manufacturing, on-ground testing, and launch of pico-class satellites. ${ }^{1-5)}$ They are a type of miniaturized can-shaped pico-class satellite platform used for technology demonstration missions. A CanSat typically has a cylinder-shaped configuration with dimensions of less than approximately $10 \mathrm{~cm}$ in radius and $20 \mathrm{~cm}$ in height, a mass of less than $1 \mathrm{~kg}$, and typically uses commercial off-the-shelf components. ${ }^{6}$ Its functionality in an extremely small package promises to yield numerous advanced technologies required for achieving challenging mission-related functions such as sub-orbital experiments, ${ }^{7}$ scientific investigations, ${ }^{8)}$ atmospheric science testing, ${ }^{9)}$ and verification of new technologies. ${ }^{10)}$ CanSats can be sent to a certain target altitude using model rockets, balloons, UAVs, airplanes, helicopters, or basically anything with flight capability. A model rocket is generally used for the actual flight test of a CanSat. A CanSat in a rocket cargo is ejected when the cargo bay opens after reaching the target altitude, and performs various scientific missions during a gliding flight using a passive or active parachute control system.

The Smart Call from the Sky (SCSky) CanSat is a picoclass satellite developed at the Space Technology Synthesis Laboratory (STSL) ${ }^{11)}$ of Chosun University, and was the Grand Prize winner at the 5th CanSat Contest hosted by the Ministry of Science, Information and Communication Technology (ICT), and Future Planning in South Korea. The starting point of the research project was to build a CanSat platform with an on-board smartphone for realizing real-time smartphone video calls between a ground station and the

(C) 2019 The Japan Society for Aeronautical and Space Sciences

*Received 18 January 2018; final revision received 26 December 2018; accepted for publication 8 February 2019.

†Corresponding author, ohu129@chosun.ac.kr
SCSky CanSat. A smartphone is the most advanced mobile operating system, combining features of a personal computer operating system with other features useful for a mobile phone. The system architecture of a smartphone is similar to that of a satellite, with the exception of the absence of propulsion and attitude control subsystems. The smartphone also contains many highly miniaturized and integrated components, such as a camera, sensors, and highly integrated computing and memory devices. The use of smartphones for the small satellite project was recently promoted not for only academic use, but also for space agencies and industries around the world as a means of space qualification of consumer-grade off-the-shelf smartphones. For example, NASA has developed a pico-class cube satellite called PhoneSat-1.0 for investigating the survivability of commercial smartphones under harsh on-orbit environments. ${ }^{12)}$ An Android operation system-based Google Nexus-one (HTC Co.) was selected as the on-board computer (OBC) of the satellite. PhoneSat-1.0 was launched in 2013 and successfully performed on-orbit operations during a designated mission life of one week. After this mission, PhoneSat 2.0 and 2.5 missions were also successfully performed. ${ }^{13-15)}$

To actualize the mission proposed, we introduced a strategy to use the characteristics of a shape memory alloy (SMA) as an actuator. The primary objective of the mission was to verify the effectiveness of a remote screen touch system actuated using an SMA wire ${ }^{16)}$ actuator, which enables remote operation of the CanSat on-board smartphone using telecommands from the ground station. The system achieves realtime streaming video calls from the on-board smartphone, allowing internal and external video images of the CanSat to be shown during actual flight. The combination of the smartphone and SMA material to realize a real-time video call has never been performed in previous CanSat missions. The additional secondary mission we performed was to acquire wide-scan images from two on-board USB cameras, 
whose elevational rotation movement was controlled using SMA spring actuators. ${ }^{17)}$

One of the most frequently used smart material-based devices with flight heritage in actual space programs is a SMA hold-and-release actuator, ${ }^{18)}$ which has been widely used for non-explosive separation mechanisms aimed at the release of mechanical constraints owing to its simplicity, reusability, high reliability against fatigue, and much lower shock levels than conventional pyrotechnic devices. The unique characteristics of the SMA wire and spring used in this study also originate in the solid-solid state phase transformation according to the operating temperature conditions. The deformed shape of an SMA at a low temperature below the martensite start temperature can be completely recovered by heating the material above the austenite finish temperature. ${ }^{19)}$ Hence, SMAs are generally used as smart actuators because they produce a high recovery force during the heating process. ${ }^{20}$ ) For example, Chiang et al. ${ }^{21)}$ developed a $2 \mathrm{U}$ cube satellite to observe the electromagnetic waves of Mercury. In this satellite, an SMA-based deployable dipole antenna 7.2-m long was applied, as shown in Fig. 3, because the space of the cube satellite to mount the antenna was strictly limited. The SMA-based antenna mechanism provided advantages including low power consumption and low shock intensity during antenna deployment. Kathryn et al. ${ }^{22)}$ undertook the mechanical design of an SMA wire-actuated prosthetic hand having five fingers with $20^{\circ}$ of freedom. The design of this prosthetic hand was based on human anatomy and actuated by SMA-based artificial muscles. The type of robotic hand described in this paper is expected to be utilized for applications requiring compact and lightweight components, such as prosthetic devices and devices for use in space and planetary exploration. In addition, the SMA actuator was applied for developing vibration isolators for space applications. ${ }^{23,24)}$ Using this approach, it is possible to build a mechanical remote screen touch system (RSTS) and wide-swath scan camera system (WSSCS) for implementing SCSky CanSat missions. In the case of the RSTS, the touch buttons integrated with SMA wires are located at designated points on the smartphone touch screen. When the ground station commands video calling to a specific phone number, each button touches the screen by the contraction and relaxation of the SMA wire according to the application of electrical current. The WSSCS is also operated in a similar manner to drive two on-board cameras for wide-swath imaging.

In this paper, SCSky CanSat development activities including the mission definition, system architecture, payloads using an SMA actuator, and validation testing at the subsystem and system levels are introduced. In addition, the effectiveness of the system demonstrated through an actual flight test is illustrated. The remainder of this paper is organized as follows. In Section 2, an overview of the SCSky CanSat system is described. In Section 3, a detailed description of the system is given. In Section 4, a subsystem design including the mission payloads is provided. In Section 5, validation of the test results, including actual flight tests is reported. In Section 6, some concluding remarks are given.

\section{Mission Objectives and System Specifications}

A CanSat mission called SCSky was developed within the framework of the CanSat competition program in Korea, pursing educational, scientific and technological purposes. The aim of this mission is to develop a smartphone CanSat with the fundamental functionality of real-time streaming video calling achieved using a RSTS and wide-scan image acquisition using WSSCS actuated using SMA actuators.

Figure 1 illustrates the architecture and operational concept of the SCSky CanSat system. The SCSky CanSat is launched using a model rocket developed by the Satellite Technology Research Center (SaTReC) at the Korea Advanced Institute of Science and Technology (KAIST). After the CanSat is released from the rocket cargo bay, the ground station sends a command with a telephone number to call the on-board smartphone during the parachute gliding flight stage of the CanSat. The RSTS, actuated using a SMA wire actuator attached to the touch screen of the smartphone, begins pressing the call number of the smartphone at the ground station. Subsequently, real-time streaming video communication between the smartphone on the ground and the one in the CanSat is realized. In addition, the on-board smartphone can also send internal and external real-time video data using the smartphone's camera switch-back function, which makes it possible to obtain both video images using only one on-board camera. The function can also be achieved using the main payload of the RSTS, and is the primary mission of this CanSat project. The secondary mission is to acquire a wide swath of images from the two on-board USB cameras, as shown in Fig. 1. The elevation angle of the camera is controlled within a range of $\pm 35^{\circ}$ through the WSSCS actuated using SMA springs.

Table 1 describes the system requirements for achieving a successful mission. There were no dedicated mission requirements from the host of the CanSat Competition because each mission was to be proposed by the respective participant. The mandatory requirements from the host of the competition were for the participants to provide status data on the CanSat obtained from attitude and position sensors during a flight demonstration.

Table 2 lists the basic specifications of the SCSky CanSat. The total mass of the CanSat including the parachute is $0.941 \mathrm{~kg}$. The CanSat is launched using a model rocket,

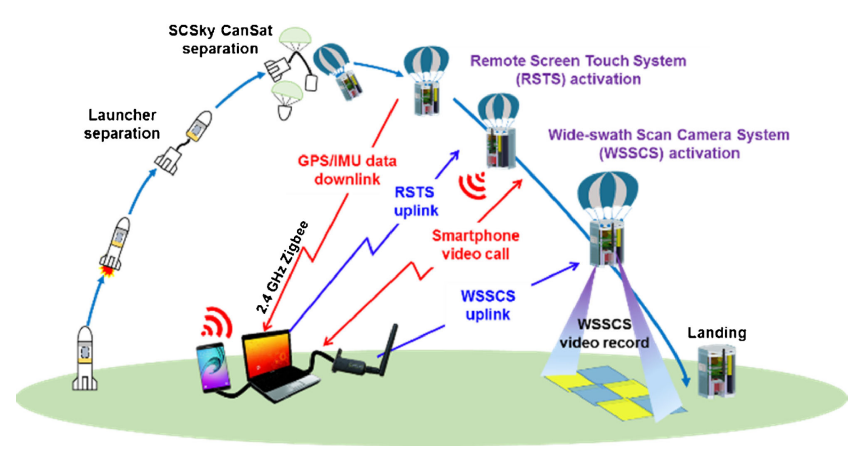

Fig. 1. System architecture and operation concept for the SCSky CanSat. 
Table 1. SCSky mission and system requirements.

\begin{aligned} & \hline ID \multicolumn{1}{c}{ Requirements } \\ & \hline 1 Total mass of the SCSky CanSat shall be less than $1 \mathrm{~kg}$. \\ & 2 $\begin{array}{l}\text { Height and diameter of the SCSky CanSat shall be less than } \\ 200 \mathrm{~mm} \text { and } 110 \mathrm{~mm} \text {, respectively, to avoid interference with the } \\ \text { cargo bay of the model rocket. }\end{array} \\ & 3 \begin{array}{l}\text { SCSky CanSat shall provide its status, including its position, } \\ \text { attitude, and illumination data during flight. }\end{array} \\ & 4 \begin{array}{l}\text { SCSky CanSat shall achieve wireless communication with the } \\ \text { ground station within a 500m range. }\end{array} \\ & 5 \begin{array}{l}\text { SCSky CanSat shall be developed within the designated } \\ \text { development schedule and costs. }\end{array} \\ & 6 \begin{array}{l}\text { No damage to the CanSat retrieved after landing shall be } \\ \text { demonstrated through a visual inspection. }\end{array} \\ & 7 \begin{array}{l}\text { SCSky CanSat shall demonstrate the success of all suggested } \\ \text { missions during flight after ejection from the rocket cargo bay. } \\ 8\end{array} \\ &$ The ground station shall send and receive all telecommands and \\ & telemetry during the flight. \\ & 9\end{aligned}

Table 2. Basic specifications of the SCSky CanSat.

\begin{tabular}{ll}
\hline \multicolumn{1}{c}{ Items } & \multicolumn{1}{c}{ Specifications } \\
\hline Name & SCSky CanSat \\
Mass & $0.94 \mathrm{~kg}$ \\
Volume (mm) & $107 \times 107 \times 156$ \\
Payloads & RSTS using SMA wire actuator \\
& WSSCS using SMA spring actuator \\
Launch method & Model rocket \\
Attitude control & Passive free-fall parachute gliding \\
OBC & MCU: ATmega2560 \\
& Memory: Flash memory 256 KB \\
Communication & Transceiver: SENA Probee-Ze10S \\
& Antenna: 1 dBi U.FL Type \\
& Frequency: 2.4 GHz \\
& Output power: 190 mAh $(627 \mathrm{Wh})$ \\
Sensors & GPS, IMU, CdS, temperature \\
\hline
\end{tabular}

and the target altitude is $300 \mathrm{~m}$. The CanSat conducts the SCSky mission during a free-fall parachute glide without the use of an attitude control system. The communication frequency used is $2.4 \mathrm{GHz}$ based on ZigBee wireless long-range communication technology. The peak consumed power of CanSat system was a maximum of $10.18 \mathrm{~W}$ in the worst case, when all of the on-board equipment is operating simultaneously. Therefore, this has a sufficient margin of $22.38 \mathrm{~W}$ with regards to the maximum available discharging power of the battery, $32.56 \mathrm{~W} .{ }^{25)}$

\section{Design Description of SCSky CanSat}

\subsection{SCSky mechanical system}

Figure 2 shows the overall mechanical configuration of the SCSky CanSat. The design is based on a can-sized platform to meet the system requirements specified in Table 1. The detailed mass budget of the SCSky CanSat is summarized in Table 3. The volume of the SCSky CanSat, with the ex-

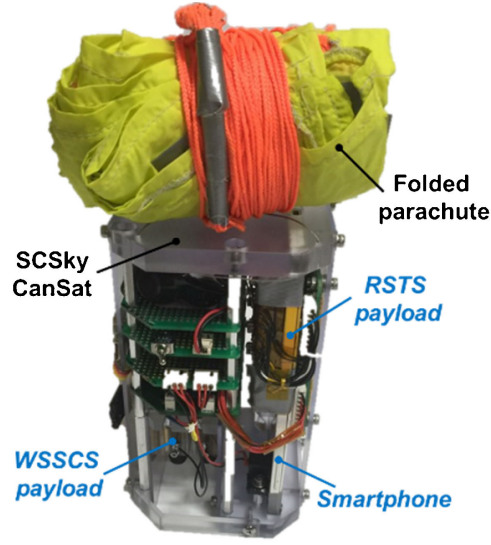

(a)

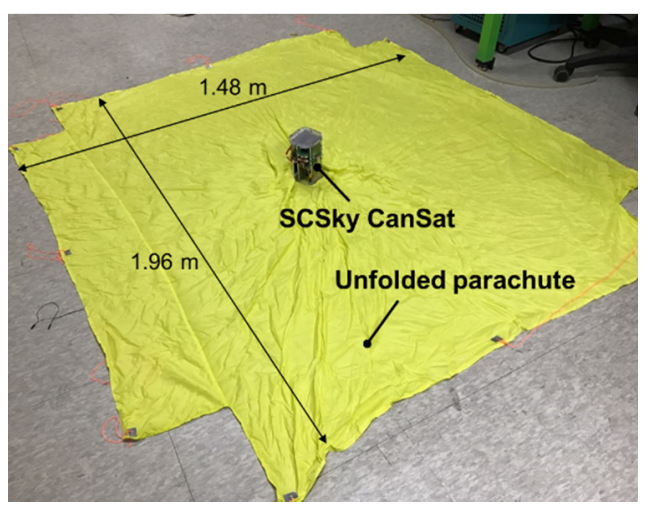

(b)

Fig. 2. Mechanical configuration of the SCSky CanSat: (a) with a folded parachute, and (b) with an unfolded parachute.

ception of the parachute, is $107 \mathrm{~mm} \times 107 \mathrm{~mm} \times 156 \mathrm{~mm}$, as shown in Fig. 2(a). All structural elements, with the exception of the electrical boards, were manufactured using polycarbonate to reduce mass.

To maximize its flight duration under a free-fall after being released from the rocket cargo bay, a cross-parachute, shown in Fig. 2(b), made using polysynthetic fiber was packed into the CanSat. This type of parachute has been widely used for CanSat applications since it is advantageous in flight stability during free-fall compared with the typical circular-shaped one. To achieve a desirable descent time for the CanSat, the area of parachute, $A$, was estimated as

$$
D=m g=\frac{1}{2} \rho V^{2} A C_{D}
$$

where, $D$ is the drag force induced by the parachute, $\rho$ is the air density, $V$ is the airspeed of the parachute, and $C_{D}$ is the drag coefficient. The values of these factors were referred to those in previous literature. ${ }^{26)}$ The estimated area of the parachute was $1.96 \mathrm{~m} \times 1.48 \mathrm{~m}$, as shown in Fig. 2. The total mass of the parachute was $0.192 \mathrm{~kg}$ including the lines connecting to the CanSat.

Figure 3 shows further details of the unit accommodation for the SCSky CanSat. The primary payload, a smartphone with an SMA wire RSTS, was installed on the $+X$ panel side and the secondary payload, a SMA spring WSSCS, was in- 
Table 3. Detailed mass budget for the SCSky CanSat.

\begin{tabular}{llc}
\hline \multicolumn{1}{c}{ Items } & & Mass $(\mathrm{kg})$ \\
\hline Structure & & 0.2 \\
Parachute & & 0.192 \\
Payloads & RSTS (Smartphone: $0.172 \mathrm{~kg})$ & 0.226 \\
& WSSCS (Camera: $0.015 \mathrm{~kg})$ & 0.08 \\
& PCB & 0.039 \\
Power module & Regulator PCB & 0.024 \\
& Battery & 0.092 \\
OBC module & Computer PCB & 0.021 \\
Comm. module & Comm. PCB & 0.005 \\
& Antenna & 0.007 \\
Sensor module & GPS, IMU, CdS & 0.053 \\
Etc. & Fastener & 0.028 \\
& Harness & 0.013 \\
\hline Total & & 0.941 \\
\hline
\end{tabular}
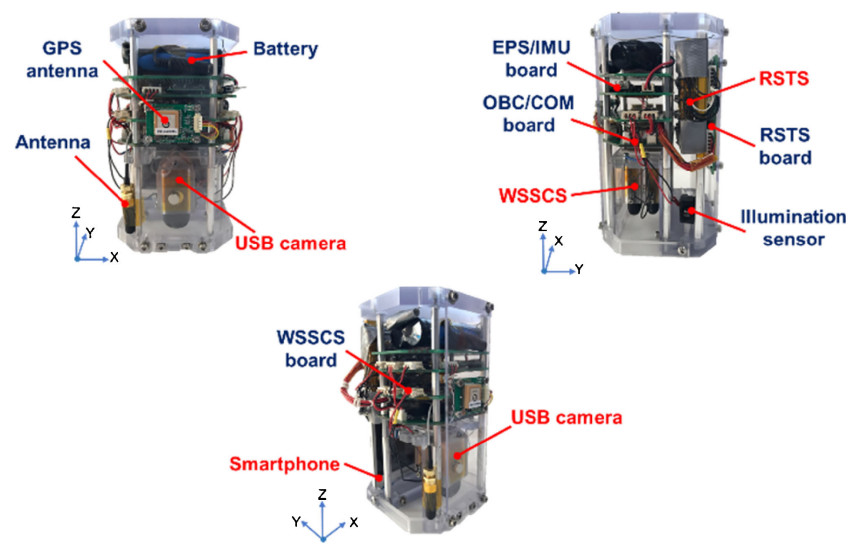

Fig. 3. Detailed unit accommodation of the SCSky CanSat.

stalled on the $-X$ and $-Z$ sides of the CanSat. The RSTS system is integrated into the touch screen of the on-board smartphone. The figure also shows that there is no mechanical constraint of the elevational rotation axis of the two USB cameras for the WSSCS, and that both sides are connected to SMA spring actuators. A more detailed description of the SCSky payloads is described in Section 3.2. The printed circuit board (PCB) stacks composed of on-board computer (OBC) module, electrical power system (EPS) module and communication system (COMS) module are positioned on the $-X$ and $+Z$ sides of the CanSat. The overall dimensions of each PCB are $86 \mathrm{~mm} \times 52 \mathrm{~mm} \times 1.6 \mathrm{~mm}$. A Li-ion battery is directly mounted to the EPS board. To maximize the accommodation efficiency, a communication antenna $(2.4 \mathrm{GHz})$ and global positioning system (GPS) antenna are installed on the $-X$ and $-Y$ sides, respectively, as shown in Fig. 3. An inertial management unit (IMU) sensor used to obtain the attitude information of the CanSat during flight is integrated directly into the EPS board. A cadmium sulfide $(\mathrm{CdS})$ illumination sensor used to check the screen status of the smartphone is installed on the $-Z$ side, near the smartphone.

\subsection{SCSky payload system}

Figure 4 shows the basic operating principle of the RSTS using a SMA wire actuator. This RSTS is mainly composed of a SMA wire actuator combined with a helical coil spring

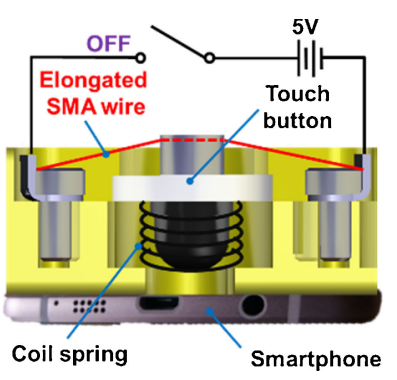

(a)

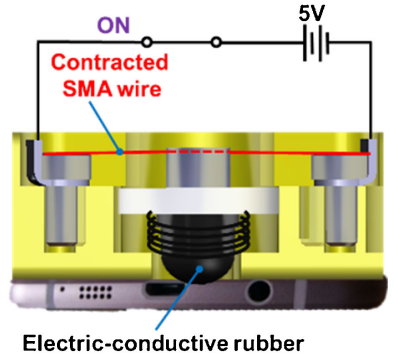

(b)
Fig. 4. Operating principle of the RSTS using a SMA wire actuator: (a) non-activation state, and (b) activated state.

Table 4. Basic specification of the SMA actuators.

\begin{tabular}{lcl}
\hline \multicolumn{1}{c}{ Items } & $\begin{array}{c}\text { SMA wire } \\
(\text { BMF-150) }\end{array}$ & $\begin{array}{c}\text { SMA spring } \\
(\text { BMX-100) }\end{array}$ \\
\hline Diameter $(\mathrm{mm})$ & 0.15 & 0.4 \\
Max. load (gf) & 144 & $10-20$ \\
Kinetic strain in length $(\%)$ & 4.0 & 200 \\
Drive current $(\mathrm{mA})$ & 340 & $100-150$ \\
Resistance $(\Omega / \mathrm{m})$ & 61 & 900 \\
Service life $($ times $)$ & $<10^{6}$ & $<10^{6}$ \\
\hline
\end{tabular}

and touch button, the tip of which is wrapped with an electrical-conductive rubber material. The SMA wire used in this study is made using a Bio-Metal Fiber series wire with a diameter of $0.15 \mathrm{~mm}$ (BMF-150, Toki Co.). ${ }^{27)}$ The basic specifications of the SMA wire used in this study are listed in Table 4. The wire is a fiber-like actuator designed to contract and extend, similar to an artificial metal-based muscle, according to the temperature when heating or cooling. The basic characteristics of the BMF series wire differ slightly from that of general SMA. Stable movement and significant selfelasticity are exhibited along the length direction because it is systematized in the direction of the fiber. The SMA wire in the RSTS becomes stiff like a piano wire and then quickly contracts, as shown in Fig. 4(b), when the temperature of the wire is increased up to $70^{\circ} \mathrm{C}$ through the application of an electric current. Under this condition, the contracted wire produces a force overcoming the axial force of the coil spring, which makes it possible to push the touch screen of the smartphone by actuating the touch button using a SMA wire responding to commands from the ground station. An input voltage of $5 \mathrm{~V}$ with a predetermined pulse-width of $130 \mathrm{~ms}$ produced by a pulse-generating flash switching device is applied to the SMA wire to prevent the wire from burning due to excessive electrical current input. When the electrical current is removed from the wire, the touch button returns to the original position through the restoration force of the coil spring; the wire becomes flexible again and stretches back to its original length, as shown in Fig. 4(a). Therefore, the touch buttons located at the designated points of the touch screen can be remotely controlled via a current input to the SMA wire using commands from the ground station. Figure 5 shows the RSTS system integrated with the on-board smartphone (Galaxy 7, Samsung Electronics). An array of 16 SMA wire touch buttons was implemented on 


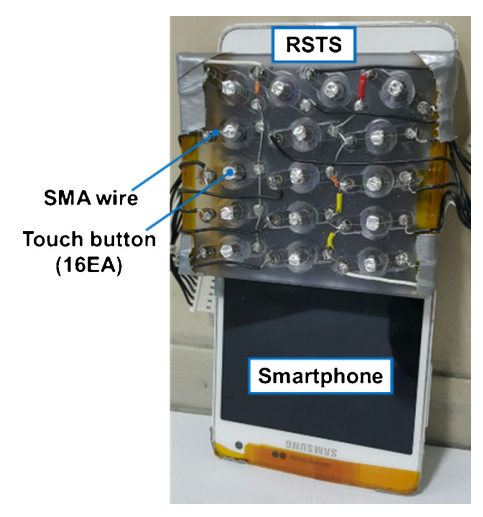

Fig. 5. Integrated RSTS on the on-board smartphone.

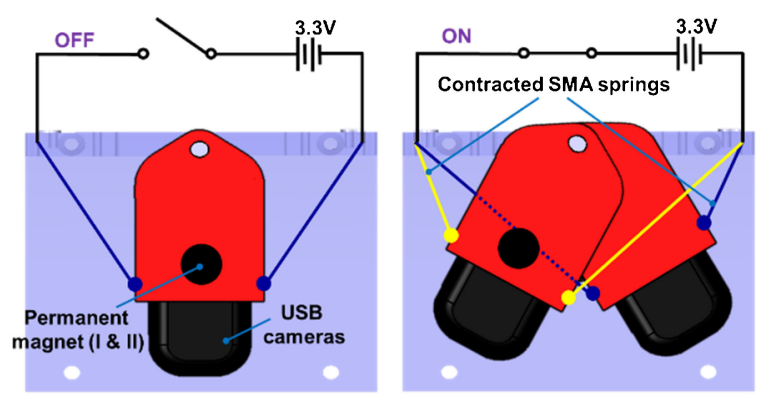

(a)

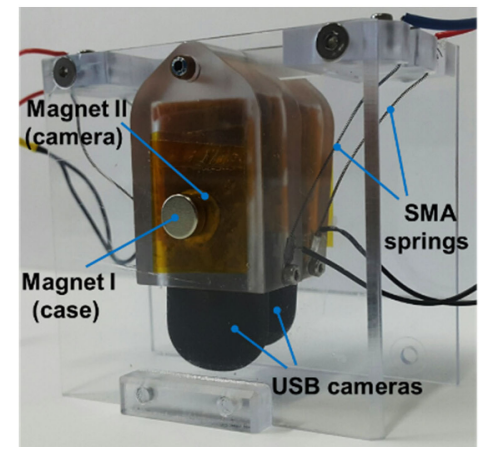

(b)

Fig. 6. WSSCS using a SMA spring actuator: (a) operating principle of the WSSCS, and (b) integrated WSSCS on the USB camera.

the RSTS, which covers the functionality of numbers from zero through nine used in phone numbers, telephone calls, and video calling, as well as switching between the rear and front camera when required for the successful execution of the SCSky mission.

Figure 6 shows the basic operating principle of the WSSCS using a SMA spring actuator. The WSSCS is mainly composed of USB cameras, SMA spring actuators, and permanent magnets. The permanent magnets installed on the camera and housing case induce electromagnetic damping that occurs due to the relative motion between the camera modules when they are excited in the rotational direction in the launch vibration environment. Therefore, this protects the camera or SMA wire by minimizing the dynamic displacement of the camera module during the CanSat launch phase. The SMA springs are connected to both sides of the camera. The SMA spring used in this study has a wide ex-

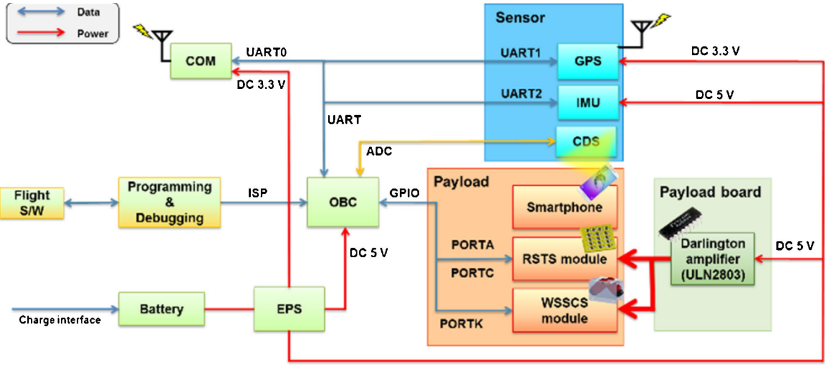

Fig. 7. Electrical system bock diagram of SCSky.

pansion and contraction range. The spring, which elongates easily at room temperature, stiffens and contracts to its original length when a current is applied. If an electrical current is reciprocally applied to each spring, a rotational movement of the camera is induced in the elevational direction. An input voltage of $3.3 \mathrm{~V}$ with a predetermined pulse-width of $500 \mathrm{~ms}$ is applied to the SMA spring. The maximum wideangle scan range of the WSSCS reflected in the design is $\pm 30^{\circ}$ owing to the spatial constraint of the CanSat. The SMA springs used in this study are Bio-Metal Helix series springs with a diameter of $0.4 \mathrm{~mm}$ (BMX-100, Toki Co.). ${ }^{27)}$ The basic specifications of the SMA spring used in this study are also listed in Table 4. A great feature of a $\mathrm{BMX}$ is that it produces a much higher force than a BMF series wire when it contracts. The dimensional change in the length direction is nearly $200 \%$ of the contracted BMX length.

\subsection{SCSky electrical system}

Figure 7 shows a block diagram of the electrical system used to achieve the mission of the SCSky CanSat. The red and blue lines in the diagram indicate power distribution and internal communications, respectively. The electrical system of the SCSky CanSat is mainly composed of payload, sensor, OBC, EPS and COMS modules. The sensor module is composed of a GPS (AKBU6, AscenKorea Co.), an IMU (EBIMU-9DOFV3, E2BOX Co.) and a CdS illumination sensor (GL5528, OEM Co.). The requirements for the GPS and IMU data acquisition during the flight were determined by the host of the CanSat competition. The CdS sensor was used to monitor the on and off status of the on-board smartphone screen. An AVR RISC-based microcontroller (ATmega 2560, ATmel Co.) was used as an OBC, which managed all electrical functions of the CanSat, such as communications control, payload operation, and telemetry data handling. The telemetry data for monitoring the health of the CanSat and mission data were sent to the COMS through UART internal communications. The OBC also controlled the SMA wire and spring actuators for operating the RSTS and WSSCS through general-purpose input/output (GPIO), which is a generic pin on an integrated circuit whose behavior, including whether it is an input or output pin, is controlled by the user during operation. The communication frequency was $2.4 \mathrm{GHz}$, and a ZigBee Pro module (SENA Co.) was used for communicating between the ground station and CanSat because this technology is intended to be simpler and 


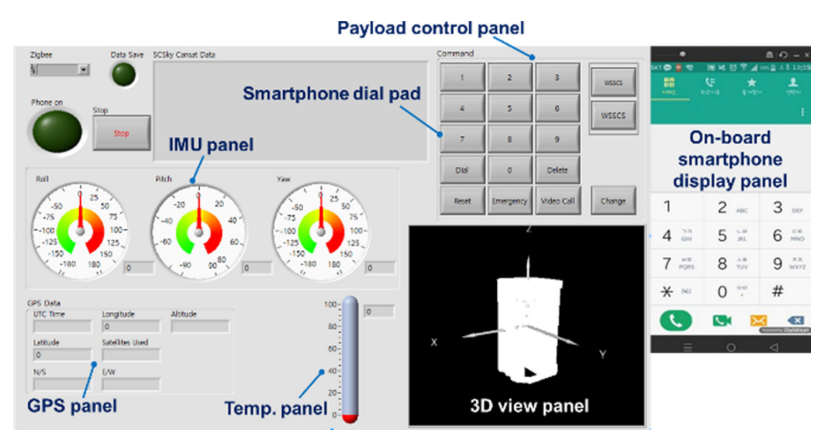

Fig. 8. Captured monitor view of the ground station.

less expensive than other wireless personal area networks, such as Bluetooth or WiFi. The EPS was comprised of a Li-ion battery $(7.4 \mathrm{~V}, 2200 \mathrm{mAh}, \mathrm{LG} \mathrm{Co}$.) and a regulator that provided specific voltages of $3.3 \mathrm{~V}$ and $5 \mathrm{~V}$ to each subsystem. In addition, a ULN2803 amplifier (Toshiba Co.) was used to amplify the low-level current from the OBC to activate the SMA wire and spring actuators.

\subsection{SCSky ground station}

The ground station used a Probee-ZE10S ZigBee Pro USB module (ProBee-ZU10, SENA Co.) for communicating with the CanSat. Figure 8 shows a captured monitor view of the ground station developed for the SCSky mission. The station was mainly composed of a payload control panel for sending telecommands to the on-board RSTS and WSSCS, IMU and GPS panels for collecting the attitude and position data of the CanSat. For real-time status monitoring of the on-board smartphone, we used a smart display panel based on the Mobizen smartphone commercial software application, ${ }^{28)}$ which enabled remote control of the smartphone via a computer through USB, WiFi or LTE connections. The Mobizen program was installed in both the smartphone and ground station. The Mobizen program made it possible to monitor the smartphone screen from the ground station in real-time. Hence, the above-mentioned mission requirement (i.e., monitor the RSTS system as to whether or not it correctly pushes the number on the smartphone) could be done. Therefore, it was possible to monitor normal operation of the RSTS in terms of whether it entered the telephone number correctly based on commands from the ground station. The wide-scan video images from the WSSCS were not directly monitored in the ground station because they were obtained from a SD card embedded in the on-board camera after completion of the CanSat flight mission.

\section{Pre-launch On-ground Validation Test}

To check the compliance of the design requirements and validate the effectiveness of the design, several pre-launch on-ground tests were conducted, from the subsystem to system levels. In this paper, payload level tests conducted to demonstrate the effectiveness of the design using the SMA smart material, and system-level tests such as a long-range communications test are described. Environmental tests such as vibration, shock, and thermal cycling tests were not con-

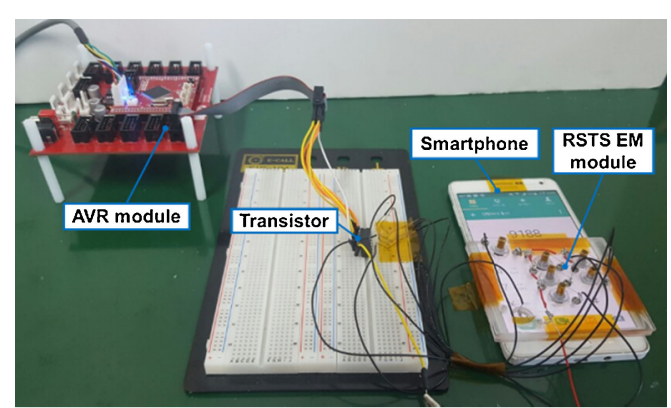

(a)

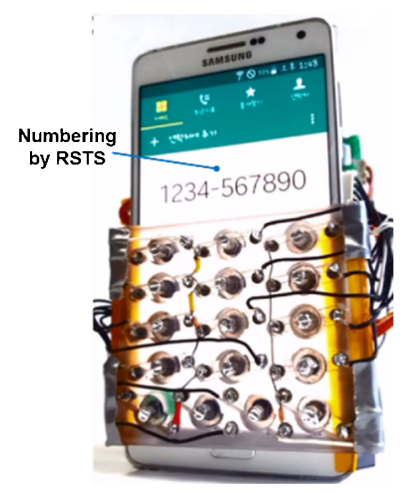

(b)

Fig. 9. RSTS on-ground test: (a) RSTS test setup, and (b) RSTS test result.

ducted during this project. However, payload functionality under the hottest temperature conditions estimated was verified because the SMA actuator performance depends heavily on the environment temperature.

Figure 9(a) shows the function test setup for verifying the engineering model (EM) of the RSTS payload conducted under ambient conditions. The RSTS mounted on the smartphone was controlled using the AVR module, and its output current amplified by a transistor was applied to the SMA wire. Figure 9(b) shows an example of the RSTS functionality test results. The test results indicate that the phone numbers of zero to nine on the touch screen of the smartphone were successfully touched through activating the SMA wires.

Figure 10 shows an example of the WSSCS functionality test results. Images of the successful camera operation sequence when activated by the SMA springs are shown in Fig. 10. The rotational movement of the camera along the elevational direction was triggered by the SMA springs when an electrical current was reciprocally applied to each spring. Figure 11 compares images captured from the USB camera with and without scanning in the elevational direction using SMA springs. The results from the WSSCS showed a much wider scene than the fixed camera without actuators.

The representative system-level tests were a function verification test under hot temperature conditions and a longrange communications test. Figure 12 shows the function test setup for hot temperature conditions. This test is important to guarantee mission success because the flight was scheduled during one of the hottest months in summer, August, in Korea. The SMA actuator temperature sensitivity 


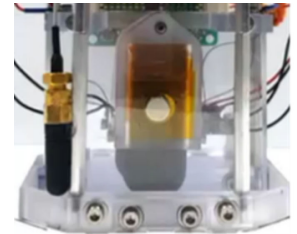

$0 \mathrm{~s}$

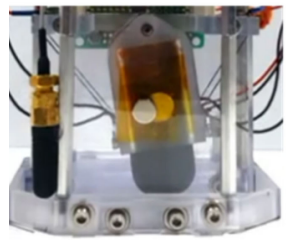

$4.0 \mathrm{~s}$

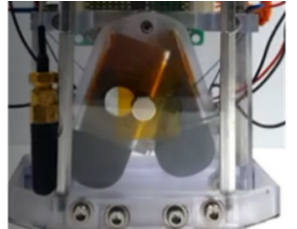

$2.8 \mathrm{~s}$

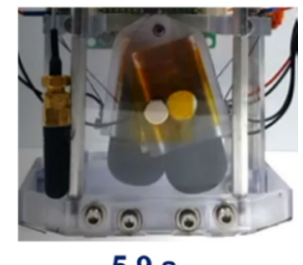

$5.9 \mathrm{~s}$

Fig. 10. Operation sequence of the WSSCS.

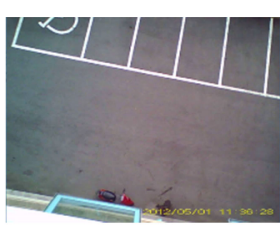

(a)
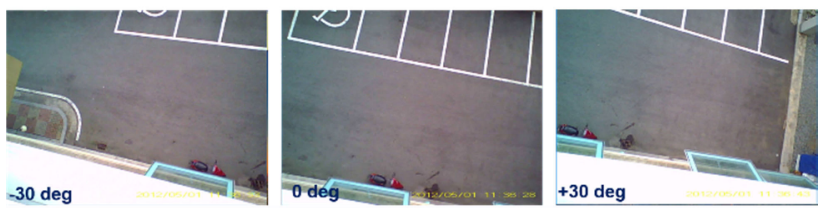

(b)

Fig. 11. Comparison of USB camera images: (a) without WSSCS, and (b) with the WSSCS

was tested because actuator performance depends heavily on the environment temperature. For example, if a variation in temperature loosens the preset tension on the RSTS SMA wires, a system failure may be induced due to a change in the wire tension boundary conditions. During the test, the CanSat was exposed outside on a rooftop. All functions of the RSTS and WSSCS combined with the ground station were successfully verified to be free of any malfunctions under temperature conditions of approximately $44^{\circ} \mathrm{C}$.

Finally, before the actual flight, we performed an end-toend far-field communications test using a test balloon system, as shown in Fig. 13. One of the main objectives of this test was to demonstrate the communication capability of the 2.4 GHz ZigBee system under conditions close to the actual flight scenario. The SCSky CanSat was raised to an altitude of approximately $100 \mathrm{~m}$ by the test balloon. The tests, including video calling from the on-board smartphone, were successfully conducted by sending mission data and housekeeping data of the SCSky CanSat to the ground station following the test scenario.

\section{Actual Launch Experiments and Results}

After being validated during on-ground testing, the SCSky CanSat achieved actual flight as the payload of a model rocket

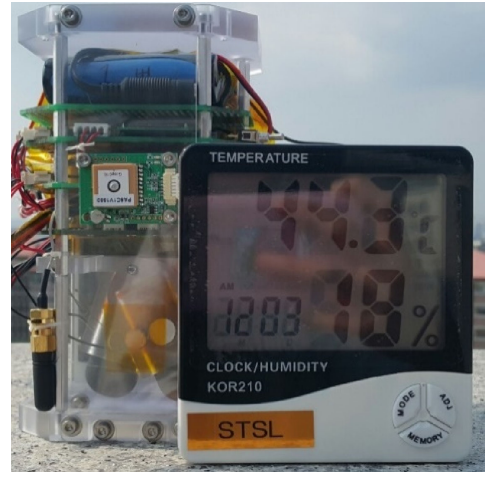

Fig. 12. System function test setup under hot temperature conditions.

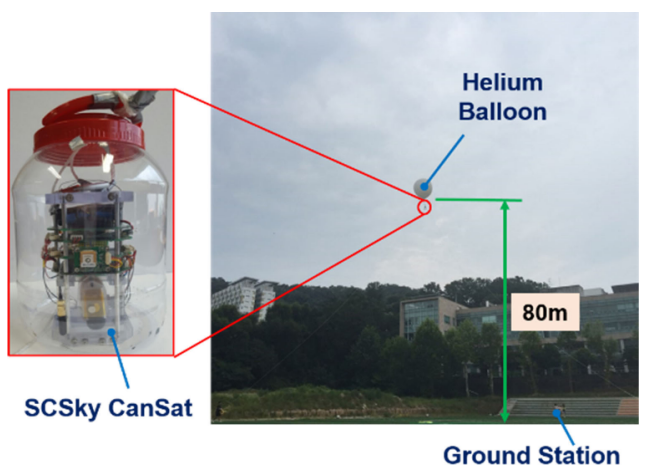

Fig. 13. End-to-end far-field communications test using a test balloon.

launched at Goheung Space Center, Korea. Separation of the CanSat from the model rocket and subsequent deployment of the parachute were successfully achieved without any problem. Figure 14 shows the status data obtained from the GPS, CdS illumination sensor, temperature sensor, and IMU as the SCSky CanSat descended. All flight status data were successfully obtained, and the results show that the CanSat separated from the rocket cargo bay at approximately 13:35 at an altitude of $230 \mathrm{~m}$. The temperature at the time of separation was $52^{\circ} \mathrm{C}$, which is $8^{\circ} \mathrm{C}$ higher than that of the $44^{\circ} \mathrm{C}$ temperature recorded during on-ground testing. Additionally, the primary mission of a real-time video call between the on-board smartphone and the ground station was successfully completed. Figure 15 shows an image of the ground station captured during the mission. The number on the right side of the screen indicates that the RSTS successfully pressed the telephone number of the smartphone on the ground following commands from the ground station. Subsequently, a real-time streaming video call between the smartphones on the ground and in CanSat was realized, as shown in the image capture in Fig. 16. The images at the lower left were from the smartphone on the ground. In addition, CanSat on-board smartphone sent internal and external real-time video data using the smartphone camera switch-back function, as shown in Figs. 16(a) and 16(b), respectively. Commands to take these photos were also sent from the ground station, actuating the RSTS to take them.

Figure 17 shows images captured using the on-board USB cameras when activated by the WSSCS. The images were 


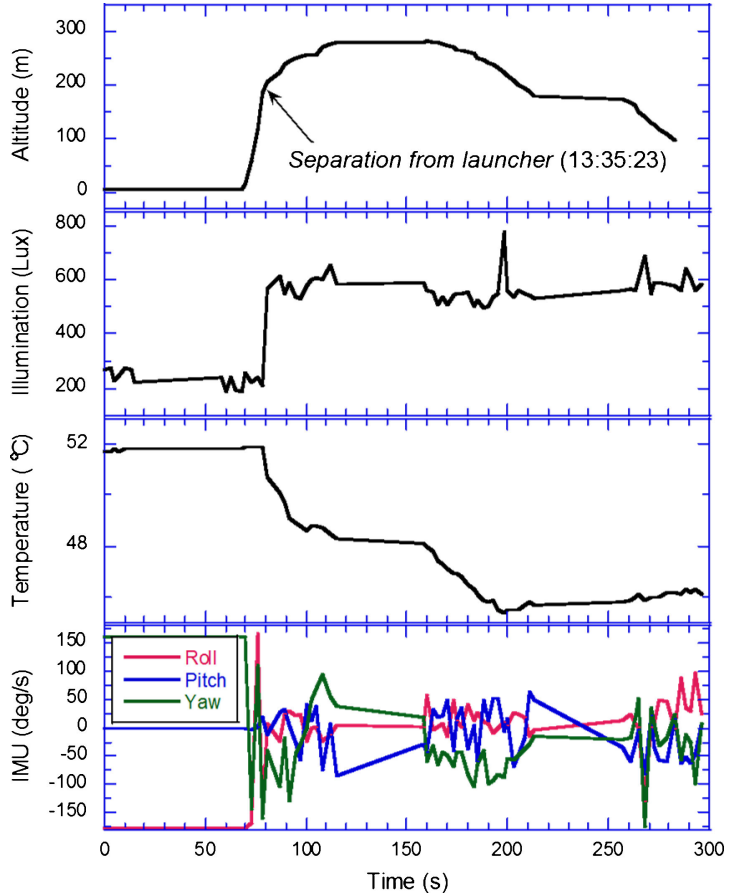

Fig. 14. Status data obtained from the GPS, CdS, temperature sensor, and IMU during the flight of the SCSky CanSat.

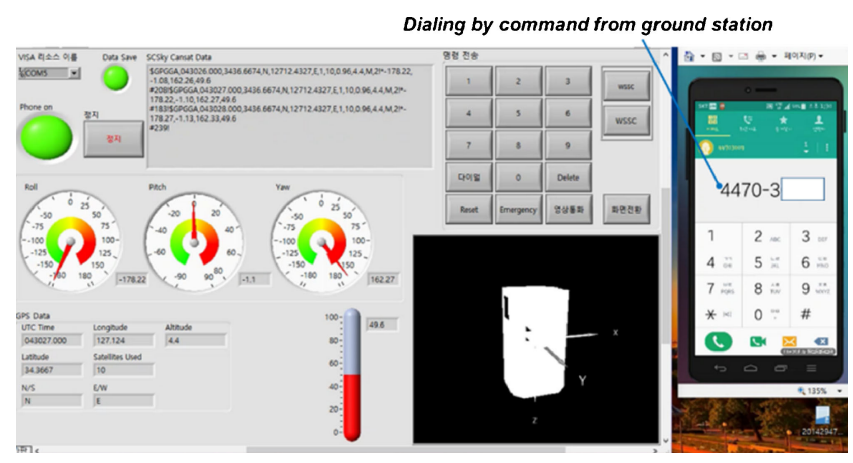

Fig. 15. Monitor view of the ground station captured during the SCSky CanSat mission.

quite unstable compared with those shown in Fig. 11, which were obtained during ground testing. This is because the attitude of the CanSat incurred substantial vibration during the flight due to a strong wind speed of $4.3 \mathrm{~m} / \mathrm{s}^{29}{ }^{29}$ The unstable attitude of the CanSat can also be seen by reviewing the IMU data provided in Fig. 14. It was determined during this project that, in order to obtain higher quality images from the WSSCS, it is necessary to isolate the camera module from the CanSat using a vibration isolation system capable of enhancing the pointing performance.

The test results of the actual launch demonstrated that the SCSky CanSat system proposed meets all of the required functions for satisfying the criteria of a successful mission, and the use of the SMA actuator was shown to be effective for realizing mission objectives.

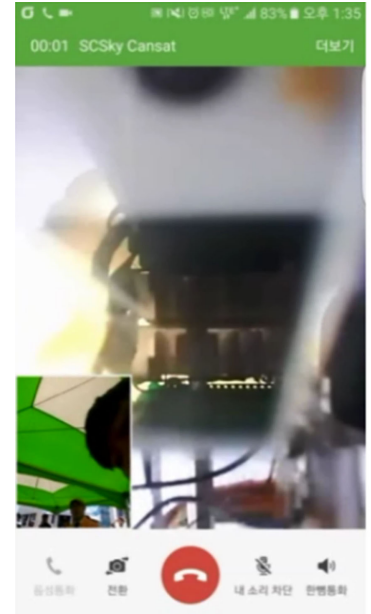

(a)

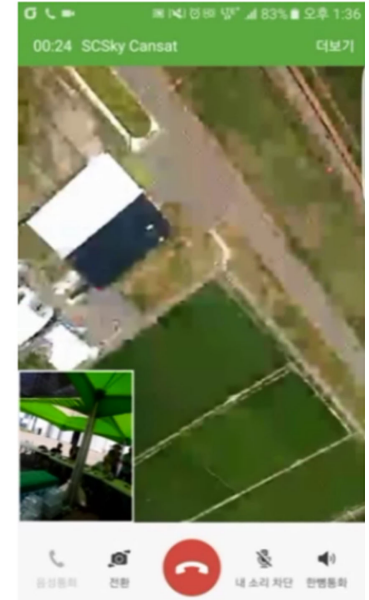

(b)
Fig. 16. Screen views of smartphone captured during the real-time video call: (a) internal view, and (b) external view.

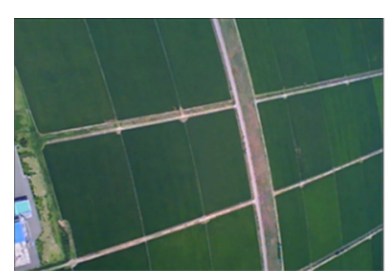

$1.03 \mathrm{~s}$

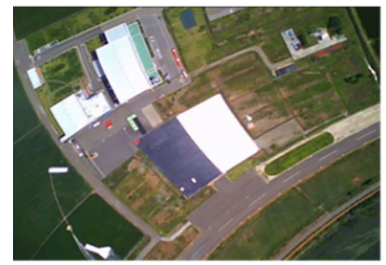

$4.26 \mathrm{~s}$

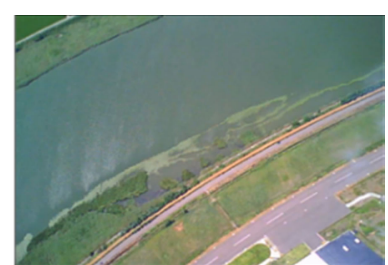

$2.61 \mathrm{~s}$

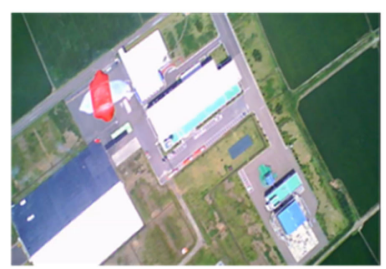

$5.34 \mathrm{~s}$
Fig. 17. Images captured from the WSSCS during flight.

\section{Conclusion}

This paper described the system design and validation of the SCSky CanSat smartphone developed to verify the technology for a remote screen touch system and wide-swath scan camera system using shape memory alloy actuators. The primary objective of the mission was to verify the effectiveness of activating a remote screen touch system using a shape memory alloy wire actuator. This system operated real-time streaming video showing the internal and external states of the CanSat through video calls during actual flight. An overview of the SCSky CanSat, including a description of its mission, was provided. Next, the SCSky CanSat design, used to guarantee the success of mission objectives by satisfying the system requirements was described. Furthermore, the effectiveness of the design and main functions of the SCSky CanSat were verified through on-ground testing before actual flight. An actual launch experiment of the CanSat using a model rocket was also conducted to validate the system. During the test, a real-time streaming video call 
between the smartphones on the ground and in the CanSat was successfully realized, as the mission intended. Wide swath images were also obtained using a wide-scan camera system, although the images were quite unstable compared with those obtained from the ground test due to the strong wind speed during the parachute gliding flight of the CanSat. The SMA material used in this study will be highly useful for space applications, especially as holding and releasing actuators for deployable structures, driving mechanisms for structural elements, and the construction of robotic arms.

\section{Acknowledgments}

This research was supported by a research fund (2017) from Chosun University.

\section{References}

1) Schilling, K.: Design of Pico-satellites for Education in Systems Engineering, IEEE Aerospace Electronic Syst. Magazine, 21 (2006), pp. 9-14.

2) Nakasuka, S.: Micro Satellite and Space Education, J. Space Technol. Sci., 16 (2000), pp. 1-8.

3) Sako, N., Tsuda, Y., Ota, S., Eishima, T., Yamamoto, T., Ikeda, I., Ii, H., Yamamoto, H., Tanaka, H., Tanaka, A., and Nakasuka, S.: Cansat Suborbital Launch Experiment-University Educational Space Program Using Can Sized Pico-satellite, Acta Astronautica, 48 (2001), pp. 767-776.

4) Celebi, M., Ay, S., Ibrahim, M. K., Aydemir, M. E., Bensaada, M., Fernando, L., Akiyama, H., and Yamaura, S.: Design and Navigation Control of an Advanced Level CANSAT, Proceedings of the 5th International Conference on Recent Advances in Space Technologies (RAST), Jun. 2011, pp. 752-757.

5) Umit, M. E., Cabanas, W., Tetlow, M., Akiyama, H., Yamaura, S., and Olaleye, S.: Development of a Fly-back CANSAT in 3 Weeks, Proceedings of the 5th International Conference on Recent Advances in Space Technologies (RAST), Jun. 2011, pp. 804-807.

6) Aly, H., Sharkawy, O., Nabil, A., Yassin, A., Tarek, M., Amin, S. M., and Ibrahim, M. K.: Project-based Space Engineering Education: Application to Autonomous Rover-back CanSat, Proceedings of the 6th International Conference on Recent Advances in Space Technologies (RAST), Jun. 2013, pp. 1087-1092.

7) Matunaga, S., Yoshihara, K., Sugiura, Y., Sekiguchi, M., Sawada, H., Tsurumi, S., Nakaya, K., Mori, M., Ui, K., Maeda, N., and Mori, O.: Titech Micro-satellite Model: CanSat for Sub-orbital Flight, Proceedings of IEEE Aerospace Conference, Mar. 2000, pp. 135-142.

8) Pande, V., Paul, J., Curillo, M., and Pallis, J.: Self Sustaining Solar Powered Cansat Exploiting the Power of the Sun, The American Society for Engineering Education (ASEE) Zone 1 Conference, Apr. 2014, pp. 1-5.

9) Bohlouri, V., Kosari, A. R., and Aliha, M. M.: Systematic Design of an Atmospheric Data Acquisition Flying Vehicle Telemetry System, Eng. Solid Mech., 2 (2014), pp. 256-276.

10) O’Mahony, C., Olszewski, O., Hill, R., Houlihan, R., Ryan, C.,
Rodgers, K., Kelleher, C., Duane, R., and Hill, M.: Reliability Assessment of MEMS Switches for Space Application: Laboratory and Launch Testing, J. Micromech. Microeng., 24 (2014), pp. 1-10.

11) Chosun University Space Technology Synthesis Laboratory, http: //www.stslab.chosun.ac.kr/

12) Salas, A. G., Attai, W., Oyadomari, K. Y., Priscal, C., Shimmin, R. S., Gazulla, O. T., and Wolfe, J. L.: PhoneSat In-flight Experience Results, The Conference of Small Satellites and Services Symposium, May 2014, pp. 1-19.

13) eoPortal Directory, https://directory.eoportal.org/web/eoportal/satellitemissions/p/phonesat-1-2 (cited on Dec. 19, 2018)

14) NASA, https://www.nasa.gov/centers/ames/engineering/projects/phonesat. html (cited on Dec. 19, 2018)

15) Spaceflight101, http://spaceflight101.com/spacecraft/phonesat-2-5/ (cited on Dec. 19, 2018)

16) Oh, H. U., Kenji, N., and Izawa, K.: Fly Wheel Vibration Isolation Test Using a Variable-damping Isolator, Smart Mater. Struct., 15 (2006), pp. 365-370.

17) Lai, Y. J., Peng, H. Y., Wu, M. W., and Shaw, J.: Research and Development of a Biomorphic Robot Driven by Shape Memory Alloys, Int. J. Mech. Aerospace Ind. Mechatronic Manufacturing Eng., 5 (2011), pp. 104-107.

18) TiNi Aerospace, http://www.tiniaerospace.com/ (cited on Dec. 19, 2018)

19) Hartl, D. J., Mabe, J. H., Benafan, O., Coda, A., Conduit, B., Padan, R., and Van Doren, B.: Standardization of Shape Memory Alloy Test Methods toward Certification of Aerospace Application, Smart Mater. Struct., 24 (2015), pp. 1-6.

20) Kohl, M., Brugger, D., Ohtsuka, M., and Takagi, T.: A Novel Actuation Mechanism on the Basis of Ferromagnetic SMA Thin Films, Sensors Actuators A Physical, 114 (2004), pp. 445-450.

21) Chiang, E. T. K., Ando, M., Tajima, K., Kitamura, K., Imai, K., and Hirakoso, N.: A Consideration of Antenna Developing Mechanism for Observation of Electric Wave from Jupiter using Cubesat, The 26th Workshop on JAXA Astrodynamics and Flight Mechanics, Jul. 2016, pp. 1-4.

22) Kathryn, J., Laurentis, D., and Mavroidis, C.: Mechanical Design of a Shape Memory Alloy Actuated Prosthetic Hand, Technol. Health Care, 10 (2002), pp. 91-106.

23) Oh, H. U., Izawa, K., and Taniwaki, S.: Development of Variable Damping Isolator using Bio-metal Fiber for Reaction Wheel Vibration Isolation, Smart Mater. Struct., 14 (2005), pp. 928-933.

24) Jeong, H. K., Han, J. H., Youn, S. H., and Lee, J. H.: Frequency Tunable Vibration and Shock Isolator using Shape Memory Alloy Wire Actuator, J. Intell. Mater. Syst. Struct., 25 (2014), pp. 1-13.

25) Kim, B. S. and Ku, C. H.: Technical Information of ICR18650 S3, LG Chem Battery Tech Center, 2007.

26) Knacke, T. W.: Parachute Recovery Systems: Design Manual, Para Pub, Jan. 1992.

27) BioMetal, http://www.toki.co.jp/biometal/ (cited on Aug. 4, 2016)

28) Mobizen, http://www.mobizen.com/ (cited on Aug. 4, 2016)

29) Korea Meteorological Administration, http://www.kma.go.kr/index.jsp (cited on Aug. 4, 2016)

Saburo Matunaga Associate Editor 